\title{
Étude de la composition minérale et des teneurs en protéines et en matières grasses de huit variétés de sésame (Sesamum indicum L.) introduites au Sénégal pour un criblage variétal
}

\author{
Birama Sene $^{1,2, *}$, Fallou Sarr ${ }^{1}$, Diegane Diouf ${ }^{2}$, Amadou Kane $^{1}$ et Djibril Traore ${ }^{1}$ \\ ${ }^{1}$ Institut de Technologie Alimentaire (ITA), Route des Peres Maristes, Hann, Dakar, BP 2765 Dakar, Sénégal \\ ${ }^{2}$ Université Cheikh Anta DIOP de Dakar, Avenue Cheikh Anta Diop, BP 5005, Dakar, Fann, Sénégal
}

Reçu le 20 février 2018 - Accepté le 30 juillet 2018

\begin{abstract}
Résumé - Huit variétés de sésame (EF153, EF147, LC162, LC164, HB168, 32-15, 38-1-7 et Birkan) qui, selon le Centre d'Étude Régional pour l'Amélioration de l'Adaptation à la Sécheresse (CERAAS), sont les mieux appréciées sur le plan agro-morphologique, ont fait l'objet de cette étude. Les teneurs en protéines, en matières grasses et en éléments minéraux de chacune d'elles ont été déterminées. L'étude des éléments minéraux a porté sur le calcium, le phosphore, le magnésium, le fer et le zinc. Les résultats ont montré que, pour les huit variétés de sésame étudiées, les teneurs en protéines varient de $22,59 \%$ à $29,37 \%$ tandis que celles en matières grasses s'établissent dans une fourchette de $48,65 \%$ à $52,45 \%$. L'étude montre aussi que toutes les variétés sont riches en éléments minéraux. Cependant, le calcium demeure l'élément le plus important dans toutes les variétés étudiées, suivi du phosphore, du magnésium, du fer et du zinc. Les meilleures teneurs minérales obtenues, pour tous les minéraux étudiés, sont avec la variété 38-1-7: magnésium $455,04 \mathrm{mg} / 100 \mathrm{~g}$; phosphore $711,17 \mathrm{mg} / 100 \mathrm{~g}$; calcium $973,22 \mathrm{mg} / 100 \mathrm{~g}$ de ; fer $10,86 \mathrm{mg} /$ $100 \mathrm{~g}$ et le zinc $7,88 \mathrm{mg} / 100 \mathrm{~g}$. L'étude statistique des teneurs en protéines, en matières grasses et en composition minérale des variétés a permis d'identifier trois variétés plus appréciées, mais aussi d'indiquer leurs domaines potentiels d'utilisation. Ainsi, les variétés LC162 et 38-1-7 pourraient être utilisées respectivement comme additifs en protéines et en éléments minéraux dans les aliments destinés aux enfants. Quant à la variété EF147, elle pourrait être recommandée pour la consommation d'huile.
\end{abstract}

Mots clés : sésame / teneur / protéines / matières grasses / minéraux / sélection variétale

\begin{abstract}
Study of mineral composition and contents about proteins and fats of eight sesame varieties (Sesamum indicum L.) introduced in Senegal for variety screening. Eight varieties of sesame (EF153; EF147; LC162, LC164; HB168; 32-15; 38-1-7; and Birkan), which are considered by the Regional Study Centre for the Improvement in Drought Adaptation (RSCIDA) as the best appreciated with regard to agro-morphological aspect, are studied. For each sesame variety, proteins, fats, and minerals contents are established. Studied minerals involve calcium, phosphorus, magnesium, iron, and zinc. Results show that, for the eight studied sesame varieties, protein's contents range from $22.59 \%$ to $29.37 \%$ whereas that of fats vary from 48.65 to $52.45 \%$. Furthermore, this study shows that all tested sesame varieties are rich in minerals. However, calcium remains the most representative of all, followed by phosphorus, magnesium, iron, and zinc. The best mineral yields come from the sesame variety 38-1-7 from which rates are as follows: magnesium, $455.04 \mathrm{mg} / 100 \mathrm{~g}$; phosphorus, $711.17 \mathrm{mg} / 100 \mathrm{~g}$; calcium, $973.22 \mathrm{mg} / 100 \mathrm{~g}$; iron, $10.86 \mathrm{mg} /$ $100 \mathrm{~g}$; and zinc, $7.88 \mathrm{mg} / 100 \mathrm{~g}$. Statistical study of contents about proteins, fats, and minerals, regarding the tested sesame varieties, permitted to identify three most appreciated sesame varieties, and also to point out their potential realms of use. Thus, LC162 and 38-1-7 varieties could be used, as protein and mineral additives respectively, in children's food. As for EF147 variety, it could be recommended for consumer oil.
\end{abstract}

Keywords: content / fats / minerals / proteins / sesame / variety screening

*Correspondance : birama. sene@ucad.edu.sn 


\section{Introduction}

Dans les pays en développement ou sous-développés, il existe un besoin urgent en produits végétaux alimentaires supplémentaires ou nouveaux pour répondre aux nombreux besoins nutritionnels ou économiques des populations. La dégradation de l'environnement agraire en Afrique, suite à la péjoration des climats vers les années 1970, a provoqué une diminution de la productivité des cultures vivrières en Afrique de l'Ouest et au Sénégal en particulier, ce qui fait perdre à l'arachide sa place de première culture de rente au Sénégal. La recherche d'alternatives aux cultures vivrières et de rente, s'impose ainsi pour assurer la subsistance des populations. La culture du sésame, connue pour ses besoins modestes en eau et en fumure (Boureima et al., 2010) et qui semblait répondre aux exigences de ce nouveau contexte, avait enregistré une forte reprise en pratique en Afrique, particulièrement au Sénégal vers les années 1985. Ainsi, ces dernières années, le sésame est de plus en plus cultivé dans la sous-région et au Sénégal en particulier, surtout dans les régions sud à sud-est du pays (Kolda, Ziguinchor et Tambacounda), du centre Nord et au Sud du bassin arachidier. Le sésame (Sesamum indicum L.), plante prometteuse de graines oléagineuses, avec un potentiel sousutilisé dans l'industrie et dans l'alimentation en Afrique (Olowe et al., 2009), se positionne comme solution à la baisse de la productivité pour contribuer à la lutte contre la pauvreté des populations rurales. Son utilisation est très importante pour l'homme. Ses graines sont non seulement essentielles pour la production de l'huile et de la pâte (tehineh), mais aussi dans la formulation des aliments tels que Halaweh (de tehineh sucré) (Abou-Gharbia et al., 2000; Kanu et al., 2007). Cependant, pour une meilleure utilisation de ses graines, des études sur sa composition biochimique s'avèrent nécessaires. Appartenant à la famille des Pedaliaceae, le sésame est une plante ancienne des cultures oléagineuses indigènes cultivé dans les pays tropicaux et subtropicaux et principalement pour ses graines. Selon Burden (2005), les graines de sésame contiennent en moyenne $50 \%$ d'huile, $25 \%$ de protéines et $15 \%$ de glucides. De fortes teneurs en huile des graines de sésame (35 à 60\%) ont été aussi rapportées par El Khier et al. (2008), Alyemeni et al. (2011), Borchani et al. (2010) et Jimoh et al. (2011). Les graines de sésame sont également source d'acides aminés essentiels et soufrés (Lee et al., 2003; Hahm et al., 2009). Elles sont riches en acides gras essentiels du groupe C18 (acides linoléique et linolénique) (Yogranjan et al., 2014). De plus, les graines de sésame contiennent de nombreux éléments minéraux et des vitamines (Kanu, 2011). Un profil minéral (mg/100g) composé de Calcium 415,38 $\pm 3,14$; Phosphore 647,25 $\pm 3,52$; Potassium 851,35 $\pm 3,44$; Magnésium $579,53 \pm 0,42$ et Sodium $122,50 \pm 4,21$ a été trouvé par Nzikou et al. (2010). Dar et Arumugam (2013) ont mis en évidence le fort plateau antioxydant du sésame avec des lignanes (sésamoline et sésamine). Il est aussi réputé d'être une source d'acides aminés essentiels et soufrés (Prakash et Naik, 2014). Selon Dang et al. (1997), les graines de sésame moulues pourraient être utilisées comme complément alimentaire contre la malnutrition infantile. Au regard de sa composition en huile, éléments minéraux, protéines, et antioxydants, le sésame (Sesamum indicum L.) est parfois considéré comme la «reine des graines oléagineuses» (Prasad, 2002; Gadade et al., 2017). Il occupe la $9^{\mathrm{e}}$ place parmi les 13 principales cultures oléagineuses qui représentent $90 \%$ de la production mondiale d'huile comestible (Adeola et al., 2010). D'ailleurs, son essor sur le marché international est dû à cette riche composition en matières grasses, en protéines, en éléments minéraux et à ses propriétés, entre autres. L'huile de sésame est aussi très riche en acides gras saturés et/ou insaturés. Afin de permettre aux producteurs de sésame de mieux valoriser leur activité, un programme de sélection variétale basé d'une part, sur les comportements agronomique et morphologique et d'autre part, sur la composition nutritionnelle pour laquelle les résultats de notre étude seront déterminants. Dans notre étude, nous avons effectué la détermination des teneurs protéiques et minéraux totaux tels que le calcium, le phosphore, le magnésium, le fer et le zinc ainsi que les teneurs en matières grasses. Le choix de ces paramètres est justifié par leur abondance et/ou leur rôle biologique essentiel dans le corps. $\mathrm{Au}$ total, les éléments minéraux représentent environ $4 \% \mathrm{du}$ poids corporel et interviennent dans une large gamme de fonctions: minéralisation, contrôle de l'équilibre en eau, systèmes enzymatiques et hormonaux, systèmes musculaire, nerveux et immunitaire (Anses, 2017; https://www.anses.fr/fr/ content/les-min\%C3\%A9raux). Cependant, pris individuellement, les minéraux choisis pour cette étude interviennent, chacun pour un bon fonctionnement de notre corps : le calcium intervient dans l'édification et le renouvellement du squelette et des dents (Brody, 1994). Il participe également à la contraction musculaire et cardiaque, à la coagulation sanguine, aux échanges cellulaires, à la perméabilité membranaire, à la libération d'hormones et à la transmission de l'influx nerveux. Le magnésium est un minéral essentiel pour l'activité enzymatique, comme le calcium et le chlorure. Il joue également un rôle dans la régulation du métabolisme glucidique et lipidique des tissus musculaires, cardiaques et nerveux et de l'équilibre acido-basique du corps. Le phosphore est nécessaire pour la croissance osseuse, la fonction rénale. Le phosphore est nécessaire pour la croissance osseuse et participe au bon fonctionnement de la fonction rénale de l'organisme. Il joue également un rôle dans le maintien de l'équilibre acidobasique du corps et participe à la plupart des réactions biochimiques de l'organisme, notamment sous forme d'adénosine triphosphate (ATP), la principale forme de stockage et de transport d'énergie dans la cellule. Quant au fer et au zinc, ils interviennent respectivement dans la fabrication et le fonctionnement de l'hémoglobine; une protéine des globules rouges qui véhicule l'oxygène depuis les poumons jusqu'aux cellules et dans la protection contre les radicaux libres et celles qui sont impliquées dans la synthèse protéique; d'où le rôle importance du zinc dans les phénomènes de renouvellement des cellules, de cicatrisation et d'immunité.

L'objectif de cette étude est de participer au programme de sélection variétale de sésame au Sénégal en déterminant la ou les variété(s) la ou les plus riche(s) en protéines, en matières grasses et en éléments minéraux pour une recommandation dans des domaines potentiels pour leur utilisation après une transformation des graines: production d'huile de sésame, enrichissement en protéines (additif) dans les formulations de farines infantiles ou complément en éléments minéraux chez les enfants. Les informations scientifiques générées dans le cadre de la présente étude seront utiles aux phytochimistes et 
nutritionnistes pour contribuer au programme de sélection variétale afin d'améliorer les attributs de la qualité du sésame.

\section{Matériel et méthodes}

\subsection{Matériel expérimental et préparation des échantillons}

Les variétés de sésame étudiées appartiennent à la collection du CERAAS. Il s'agit de la «32-15» qui est issue d'un croisement de deux variétés, San Mario (Argentine) X Satara Branco (Brésil) et du «Birkan» issu d'un cultivarmutant turc par rayons gamma de la variété Muganli-57; deux parents homologués au Sénégal qui nous viennent respectivement du Burkina Faso et de la Turquie, et des mutants «EF147, LC162, EF153 et LC164» plus deux autres variétés «HB-168 et 38-1-7», toutes, testées et appréciées en station et au champ au Sénégal. Les graines de sésame de ces variétés ont été nettoyées manuellement pour enlever celles endommagées. Ensuite, les graines nettoyées ont été triées et broyées puis stockées dans des sachets plastiques jusqu'à leur utilisation.

\subsection{Analyses chimiques}

Les procédures standards ou les méthodes décrites dans AOAC (2007) ont été utilisées. Le nombre de répétions était de 4 pour les matières grasses et 6 pour les protéines et pour tous les minéraux étudiés.

Le magnésium $(\mathrm{mg} / 100 \mathrm{~g})$ a été déterminé après digestion de l'échantillon (environ $1 \mathrm{~g}$ ) en utilisant un spectrophotomètre d'absorption atomique (SAA) à $285,2 \mathrm{~nm}$. La teneur en fer a été estimée à partir de la courbe d'étalonnage standard $(0,125-$ $0,25-0,5 \mathrm{mg}$ Fer / 1) préparée à partir de solutions certifiées à $1000 \mathrm{ppm}$ et en utilisant de l'air-acétylène comme source de flamme pour l'atomisation.

Le phosphore est déterminé sous forme de phosphate par la méthode spectrophotométrique à UV-visible, au moyen du vanadium phosphomolybdate (vanadate). Le phosphore présent sous sa forme orthophosphate $\left(\mathrm{PO}_{4}{ }^{3-}\right)$, réagit avec le réactif vanadate-molybdate pour produire un complexe jaune-orangé, dont l'absorbance est mesurée à une longueur d'onde de $470 \mathrm{~nm}$. La teneur en phosphore est ensuite déterminée selon la courbe d'étalonnage standard (0-2,5-5-10-20-30-40-50 mg P / 1) préparée en fonction de la concentration des étalons.

Le fer $(\mathrm{mg} / 100 \mathrm{~g})$ a été déterminé après digestion de l'échantillon (environ $1 \mathrm{~g}$ ) en utilisant un spectrophotomètre d'absorption atomique (SAA) à $248,3 \mathrm{~nm}$. La teneur en fer a été estimée à partir de la courbe d'étalonnage standard $(1,25-$ 2,5-5,0 mg Fer / 1) préparée à partir de solutions certifiées à $1000 \mathrm{ppm}$ et en utilisant de l'air-acétylène comme source de flamme pour l'atomisation

Le calcium $(\mathrm{mg} / 100 \mathrm{~g})$ a été déterminé après digestion de l'échantillon (environ $1 \mathrm{~g}$ ) par un spectrophotomètre d'absorption atomique (SAA) à $422,7 \mathrm{~nm}$. La teneur en calcium a ensuite été estimée à partir de la courbe d'étalonnage standard (1-2-4 mg Ca/ 1) préparée à partir de solutions certifiées à $1000 \mathrm{ppm}$ et en utilisant de l'air-acétylène comme source de flamme pour l'atomisation

Le zinc $(\mathrm{mg} / 100 \mathrm{~g})$ a été déterminé après digestion de l'échantillon (environ $1 \mathrm{~g}$ ) par un spectrophotomètre d'absorption atomique (SAA) à $213,9 \mathrm{~nm}$ en utilisant de l'air-acétylène comme source de flamme pour l'atomisation. Le niveau de zinc a ensuite été estimé à partir de la courbe d'étalonnage standard $(0,25-0,50-1,0 \mu \mathrm{g} \mathrm{Zn} / \mathrm{ml})$ préparée à partir de solutions certifiées à $1000 \mathrm{ppm}$ et en utilisant de l'air-acétylène comme source de flamme pour l'atomisation

La teneur en protéines brutes del'échantillon (environ $0,2 \mathrm{~g}$ ) a été évaluée par la méthode de Kjeldahl avec un minéralisateur BUCHI Switzerland (kjeldigesteur K-449) et un distillateur kjelflex K-360). La teneur en azote multipliée par le facteur de conversion de l'azote en protéines $(6,25)$ donne la teneur en protéines.

La matière grasse brute $(\%)$ a été déterminée par extraction au soxtherm Gerhard, de l'échantillon (environ $1 \mathrm{~g}$ ) en utilisant de l'hexane comme solvant.

\subsection{Analyse statistique}

Des données en quintuplé ont été soumises à ANOVA en utilisant le système d'analyse statistique (XLSTAT) version 6.0. Les différences significatives entre les moyennes ont été déterminées par les tests à plages multiples de Turkey au seuil de $5 \%$.

\section{Résultats et discussion}

\subsection{Caractéristiques physiques des graines de sésame}

La couleur des graines des huit génotypes ont été examinée par simple observations visuelles. Les graines de ces génotypes présentaient des couleurs variables allant du blanc, brun clair au brun. Ces variations de couleur ont été rapportées plus tôt par Gandhi et Srivastava (2007) puis par Mohammed et Hamza (2008).

\subsection{Composition nutritionnelle}

\subsubsection{Composition minérale}

Le tableau 1 ci-dessus présente les teneurs en matière sèche des graines en éléments minéraux $(\mathrm{Mg}, \mathrm{P}, \mathrm{Ca} ; \mathrm{Fer}$ et $\mathrm{Zn})$ des différentes variétés de sésame étudiées. Il ressort de ce dernier que les graines des variétés de sésame étudiées contiennent une quantité importante de minéraux. La concentration moyenne en $\mathrm{mg} / 100 \mathrm{~g}$ de matière sèche du calcium $(630,42)$ est plus élevée, suivie de celle du phosphore $(603,74)$, du magnésium $(390,59)$, du fer $(10,50)$ et en fin du zinc $(6,54)$. La composition minérale $(\mathrm{Ca}, \mathrm{P}, \mathrm{Mg}, \mathrm{Fer}, \mathrm{Zn})$ des différentes variétés de sésame a montré des variations significatives.

Leur choix se justifiait par leurs rôles dans l'organisme mais il existe d'autres minéraux. Parmi les macro-minéraux analysés, le calcium était prédominant suivi du phosphore et ensuite vient le magnésium. Cet ordre de prédominance des teneurs corrobore les études de Deosthale (1981), Elleuch et al. (2007), Kanu (2011) et de Deme et al. (2017). Par contre, pour les deux microéléments minéraux analysés, le fer a donné la valeur moyenne la plus élevée $(15,63 \pm 2,58 \mathrm{mg} / 100 \mathrm{~g})$ avec la variété EF153, suivi du zinc avec $7,88 \pm 0,40 \mathrm{mg} / 100 \mathrm{~g}$ pour la variété 38-1-7. Les teneurs en fer et en zinc, obtenues dans cette étude sont en accord avec les travaux rapportés par Deme et al. (2017). Des teneurs similaires ont été aussi rapportées par Sene et al. (2017) sur une variété très prisée au Sénégal avec la 
Tableau 1. Teneur en pourcentage pondéral $(\mathrm{mg} / 100 \mathrm{~g})$ de matière sèche des graines en éléments minéraux $(\mathrm{Mg}, \mathrm{P}, \mathrm{Ca} ; \mathrm{Fer}$ et $\mathrm{Zn})$ des différentes variétés de sésame. Les moyennes d'une même colonne suivies d'une même lettre ne sont pas statistiquement différentes $(\alpha \leq 0,05)$.

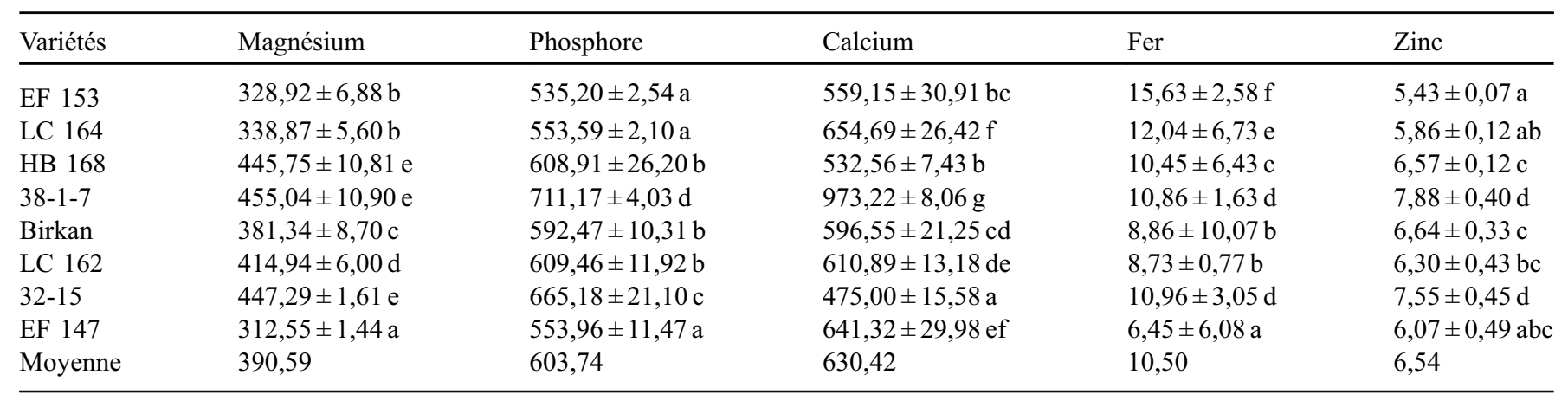

Tableau 2. Teneurs en protéines et matières grasses des différentes variétés de sésame comparées à celles de la littérature.

\begin{tabular}{lllll}
\hline Variétés & Protéines (\%) & Matières grasses $(\%)$ & Protéines rapportées(\%) & M grasses rapportées(\%) \\
\hline EF153 & $25,26 \pm 0,42 \mathrm{~b}$ & $51,74 \pm 0,74 \mathrm{~cd}$ & $22 \pm 1,5$ (Gharby et al., 2015) & $52 \pm 2,5$ (Gharby et al., 2015) \\
LC164 & $25,66 \pm 0,61 \mathrm{~b}$ & $50,22 \pm 1,02 \mathrm{abc}$ & $34,4 \pm 0,8$ (Sabah El et al., 2008) & $47,4 \pm 0,4$ (Sabah El et al., 2008) \\
HB168 & $26,92 \pm 0,25 \mathrm{c}$ & $49,78 \pm 0,29 \mathrm{abc}$ & $20 \pm 0,12$ (Nzikou et al., 2009) & $54 \pm 0,16$ (Nzikou et al., 2009) \\
$38-1-7$ & $22,59 \pm 0,07 \mathrm{a}$ & $49,46 \pm 1,04 \mathrm{ab}$ & $19 \pm 1$ (Ogbonna et Ukaan, 2013) & $36 \pm 5$ (Ogbonna et Ukaan, 2013) \\
Birkan & $26,67 \pm 0,34 \mathrm{c}$ & $50,92 \pm 1,19 \mathrm{bcd}$ & $21 \pm 0,1$ (Unal et Yalcsn, 2008) & $54,3 \pm 1$ (Unal et Yalcsn, 2008) \\
LC162 & $29,37 \pm 0,18 \mathrm{~d}$ & $48,65 \pm 0,47 \mathrm{a}$ & $18,92-23$ (Hassan, 2012) & $56-60$ (Hassan, 2012) \\
$32-15$ & $25,74 \pm 0,27 \mathrm{~b}$ & $51,12 \pm 1,4 \mathrm{bcd}$ & 24,63 (Borchani et al., 2010) & 49,7 (Alyemeni et al., 2011) \\
EF147 & $25,39 \pm 0,55 \mathrm{~b}$ & $52,45 \pm 0,59 \mathrm{~d}$ & 21,78 (Jimoh et al., 2011). & 43,3 Gopalan et al., (2011) \\
\hline
\end{tabular}

$\mathrm{M}=$ matières.

même tendance de variation des teneurs, faisant du calcium l'élément minéral majoritaire, suivi du phosphore et du magnésium. De toutes les variétés étudiées, 38-1-7 a la plus forte teneur en calcium $(973,22 \pm 8,06)$ et la plus faible $(475,00 \pm 15,58)$ était trouvée sur la variété $32-15$. Les teneurs en calcium de cette étude sont inférieures à celles rapportées par Sher et al. (2010) et par Zebib et al. (2015) qui ont trouvé, respectivement $1450 \mathrm{mg} / 100 \mathrm{~g}$ et 1225,$71 ; 1172,08 \mathrm{mg} / 100 \mathrm{~g}$ dans certaines variétés de sésame indiennes et dans des cultivars éthiopiens. Une teneur plus faible en Ca (228,3 mg/ $100 \mathrm{~g})$ a été rapportée chez certains cultivars libanais par Pellett et Shadarevian (1970) cités par Alyemeni et al. (2011). De même, Nzikou et al. (2010) ont obtenu des teneurs de calcium $(415,38 \mathrm{mg} / 100 \mathrm{~g})$ inférieures aux nôtres.

Les teneurs en phosphore varient entre 535,20 (EF153) et $711,17(38-1-7) \mathrm{mg} / 100 \mathrm{~g}$ chez toutes les variétés étudiées. Cette fourchette de teneurs en phosphore est en-deçà des résultats de Deosthale (1981) sur certains cultivars indiens qui ont donné de teneurs plus élevées $(872 \mathrm{mg} / 100 \mathrm{~g})$. Comparées aux résultats de teneurs (540 à $640 \mathrm{mg} / 100 \mathrm{~g}$ ) d'Alyemeni et al. (2011) sur des cultivars saoudiens, nos variétés affichent une meilleure composition en phosphore mais confirment les teneurs trouvées par Nzikou et al. (2010) (647,25 mg/100 g).

Nous avons noté que les valeurs obtenues pour le magnésium $(312,55$ à $447,29 \mathrm{mg} / 100 \mathrm{~g})$ sont plus élevées que celles rapportées par Alyemeni et al. (2011) sur des variétés saoudiennes et indiennes et plus faibles que celles $(579,53 \mathrm{mg} / 100 \mathrm{~g})$ de Nzikou et al. (2010); cette variabilité de teneurs de ces macroéléments pourrait être attribuée à des combinaisons de facteurs environnementaux et génétiques ainsi qu'aux méthodes utilisées pour l'analyse. Les résultats ont montré qu'entre les variétés, et selon l'élément minéral, quelques ressemblances de teneurs sont notées dans la quantification de minéraux. Ce qui semble indiquer des relations de parenté entre les variétés. Cette ressemblance est notée entre les deux mutants (EF153 et LC164) de la variété de 32-15 pour les teneurs en magnésium et en phosphore et entre les variétés HB168 et 38-1-7 pour la teneur en magnésium. Il est aussi important de noter la différence des teneurs non significative entre les deux parents $32-15$ et $38-1-7$ pour les trois éléments minéraux que sont le magnésium, le fer et le zinc (Tab. 1). Le calcium et le magnésium jouent un rôle important dans la photosynthèse, le métabolisme des hydrates de carbone, des acides nucléiques et des agents de liaison des parois cellulaires (Russell, 1973).

\subsubsection{Teneur proximale en protéines et en matières grasses des graines de variétés de sésame}

Le tableau 2 présente les résultats de la composition en protéines et en matières grasses brutes des graines des variétés de sésame soumises à notre étude. Il ressort de cette étude que la teneur en protéines dans les graines entières des variétés de sésame varie de $22,59 \%$ à $29,37 \%$ avec une valeur moyenne de 25,95\%. La variété LC162-1 présente la meilleure teneur en protéines $(29,37 \%)$ et la variété $38-1-7$, la plus faible teneur $(22,59 \%)$. 
Cette variation des teneurs en protéines est généralement attribuée aux facteurs génétiques et/ou environnementaux ou à la méthode d'analyse. Toutefois, la fourchette des teneurs en protéines dans nos échantillons de variétés est plus large que celle rapportée par Kahyaoglu et Kaya (2006) et Adebowale et al. (2011) et dont les valeurs sont respectivement (16,20\% à $26,29 \%)$ et $(12 \%$ à $23,0 \%)$. Une gamme de teneurs en protéines de $19 \%$ à $25 \%$ qui est en-dessous de nos résultats a été aussi trouvée par Yahya (1998) avant d'être confirmée par celle $(19,81 \%$ à 24,45\%) d'Ozkan et al. (2012) sur des variétés de sésame turques. Comparées aux résultats (32 à $40 \%$ ) trouvés par El Khier et al. (2008), nos variétés de sésame présentent des teneurs en protéines plus faibles mais donnent des teneurs supérieures à celles mesurées par Unal et Yalcsn (2008) ; Hassan (2012) et Gharby et al. (2015). Nos résultats sont aussi inférieurs au contenu de protéines trouvé par Orruno et Morgan (2007) et à celui rapporté par Kim et al. (2014) sur des variétés de sésame noir et blanc. En revanche, la moyenne brute des teneurs en protéines $(25,95 \%)$ obtenue dans cette étude est également supérieure aux valeurs moyennes de $24,63 \%, 21,78 \%$ et 20,00\% rapportées respectivement par Borchani et al. (2010), Jimoh et al. (2011) et Nzikou et al. (2010). Prenant seule la variété LC162, qui donne la meilleure teneur en protéine de notre étude, elle affiche une teneur en protéines supérieure à celles des cultivars de sésame analysés dans d'autres pays comme au Maroc $22 \%$, au Congo $20 \%$, au Nigéria 19\%, en Turquie $21 \%$ et en Égypte 18,93\% (Gharby et al., 2015). Sa teneur en protéines est aussi au-dessus de celles de l'arachide qui donne $27,50 \%$ en moyenne et du niébé qui fournit $19,90 \%$ de protéines. Cette particularité nous amène à la recommander dans ce programme de sélection et plus spécifiquement pour l'enrichissement en protéines (additif) dans les farines infantiles. Il est évident que la protéine est l'un des nutriments qui sont souvent faibles dans les produits végétaux.

Étant donné qu'une teneur élevée en huile enregistrée dans les cultivars marocains (plus de $50 \%$ ) est un trait souhaitable pour les programmes de sélection visant à améliorer les cultivars de sésame, certaines de nos variétés étudiées EF153, 32-15 et EF147 méritent une sélection. Elles ont des teneurs en matières grasses supérieures à $50 \%$. En outre, il existe une différence significative entre les variétés concernant la teneur en matières grasses : elles varient de $48,65 \%$ à $52,456 \%$ avec une moyenne de $50,548 \%$. Cette variabilité de la teneur en huile ne peut être attribuée qu'aux facteurs variétaux, environnementaux, à l'interaction des deux facteurs, à la date de récolte des graines ou à la méthode d'analyse. La meilleure teneur est observée avec la variété EF147 tandis que la variété LC162 affiche la plus faible teneur en matières grasses. Les résultats de la présente étude sont conformes aux conclusions de Mohammed et Hamza (2008), de Nzikou et al. (2009) et d'Asghar et Majeed (2013). Des résultats similaires (49,7\%) ont été rapportés par extraction à partir de graines de sésame par Alyemeni et al. (2011) et Gadade et al. (2017). Ils corroborent les résultats de Bahkali et al. (1998) et de Dernekbasi et al. (2017) qui rapportent une composition respective de $43,2 \%$ à $54,0 \%$ et 47,8 à $52,2 \%$ d'huile dans des variétés turques. Ils sont aussi en accord avec la moyenne de teneurs $(50 \%)$ trouvée par Kanu et al. (2007) mais également corroborent les conclusions en contenu d'huile (51\%) des variétés de sésame étudiées par Peter (2004). D'autres études menées par Zebib et al. (2015) sur des variétés égyptiennes ont donné des résultats similaires $(50,88 \% ; 52,67 \% ; 51,18 \%)$ pour les matières grasses.

Cependant, les résultats de cette étude diffèrent de ceux de Makinde et Akinoso (2013 et 2014) sur des variétés nigérianes qui ont donné une moyenne de 46,09 $\pm 0,04 \%$ de teneurs en matières grasses. Comparées aux résultats de Gopalan et al. (2011) et de Bukya et Vijayakumar (2013) qui ont trouvé respectivement $43,30 \%$ et $44,53 \%$ de matières grasses sur des graines entières de variétés de sésame en Inde, nos variétés donnent, toutes, des teneurs au-delà de leurs résultats. Cependant, Hassan (2012) a rapporté une teneur en huile de cultivars de sésame de $56 \%$. D'autres moyennes de teneurs en huile significativement plus élevées $(63,25 \%)$ ont été rapportées chez les cultivars turcs (Baydar et Turgut, 1999). Étant donné que la température influence la teneur en huile, Rondanini et al. (2003) ont rapporté qu'un stress hydrique précoce entraîne une réduction de la teneur en huile et lorsqu'il est tardif, il induit une augmentation de la teneur en huile. Cependant, selon El Asri et al. (2000) puis Flagella et al. (2000), un stress modéré après écoulement a toujours entraîné une augmentation de la teneur en huile. Par ailleurs, comparée aux teneurs habituelles d'huile de l'arachide (47,60\%) et du soja $(20 \%)$, la variété EF 147 qui est la plus riche en matières grasses $(52,47 \pm 0,60)$ de toutes les variétés de sésame étudiées, donne une teneur en matières grasses plus élevée. Par conséquent, elle est recommandée dans ce programme de sélection pour une production d'huile par une transformation de ses graines. Sa sélection dans ce programme pourrait impacter directement le niveau des revenus des populations qui sont dans la filière sésame. Comparées à d'autres spéculations comme le niébé et l'arachide, bien prisés, pour leur composition respective en protéines et en matières grasses, les variétés LC 162 et EF 147 donnent respectivement des teneurs en protéines $(29,37 \pm 0,18 \%)$ et des teneurs en matières grasses $(52,45 \pm 0,59 \%)$ plus importantes par rapport aux variétés $81 \mathrm{D}$ et $87 \mathrm{D}$ de niébé et aux variétés A26 et A32 de l'arachide. Selon Nwaga et al. (2000), les variétés de niébé $81 \mathrm{D}$ et $87 \mathrm{D}$ ont donné respectivement $24,34 \%$ et $29,21 \%$ de protéines et les variétés d'arachide A26 et A32 $(27 \%$ et $49,50 \%$ ) de matières grasses. L'étude corrélative de teneurs en protéines et en matières grasses montre que plus une variété est riche en protéines, moins bonne est sa teneur en huile.

\section{Conclusion}

Cette étude montre que les graines de variétés de sésame de notre collection représentent une bonne source de protéines et de matières grasses brutes, mais aussi d'éléments minéraux comparées aux autres variétés de sésame cultivées dans le monde. Les variétés LC 162 et EF 147 présentent respectivement de très bonnes compositions protéiques et en matières grasses par rapport à toutes les variétés étudiées alors que la meilleure teneur en minéraux était obtenue avec la variété 38-1-7. Par conséquent, elles pourront être sélectionnées dans ce programme d'amélioration mais aussi pour la valorisation du sésame respectivement dans l'enrichissement en protéines des farines infantiles, dans la production d'huile et pour un complément en éléments minéraux. Cependant, pour une utilisation nutritionnelle plus efficace, il serait nécessaire de 
procéder à des traitements qui réduisent la teneur en acide phytique de la graine. En plus, l'utilisation des graines de sésame comme aliment fonctionnel pour la nutrition humaine devrait être réalisée en mélange avec d'autres fruits concentrés.

\section{Perspectives pour une étude plus exhaustive}

$\mathrm{Au}$ vu des teneurs obtenues dans cette étude sur certaines variétés et l'importance de ces nutriments dans l'organisme, il serait important de poursuivre l'étude afin de mesurer la biodisponibilité de ces éléments minéraux, en déterminant la composition des acides aminés essentiels et en étudiant le profil des acides gras de toutes les variétés. Cela permettrait une caractérisation plus complète et donc une recommandation précise pour leur sélection dans le cadre d'un programme d'amélioration et de redynamisation de la filière sésame.

\section{Références}

Abou-Gharbia HA, Shehata AAY, Shahidi F. 2000. Effect of processing on oxidative stability and lipid classes of sesame oil. Food Res Int 33: 331-340.

Adebowale AA, Fetuga GO, Falore OA, Adegunwa MO, Sanni SA. 2011. Varietal characterization of the physical properties, proximate and mineral composition of improved sesame (Sesamum indicum) seeds. J Nat Sci Eng Technol 10(2): 10-22.

Adeola YB, Augusta CO, Oladejo TA. 2010. Proximate and mineral composition of whole and dehulled Nigerian sesame seeds. Afr $J$ Food Sci Technol 1(3): 71-75.

Alyemeni MN, Basahy AY, Sher H. 2011. Physico-chemical analysis and mineral composition of some sesame seeds (Sesamum indicum L.) grown in the Gizan area of Saudi Arabia. $J$ Med Plants Res 5(2): 270-274.

Anses. 2017. Les minéraux: présentation et rôle des matières minérales dans l'organisme. Disponible sur: https://www.anses. $\mathrm{fr} / \mathrm{fr} /$ content $/$ les-min $\% \mathrm{C} 3 \% \mathrm{~A} 9 \mathrm{raux}$.

AOAC. 2007. Officials methods of analysis of AOAC International, 18th ed, 2005, Rev 2007. Arlington, VA, USA: Association of Official Analytical Chemists.

Asghar A, Majeed MN. 2013. Chemical characterization and fatty acid profile of different sesame verities in Pakistan. Am J Sci Indus Res. ISSN: 4.6:540-545.

Bahkali AH, Hussain MA, Basahy AY. 1998. Protein and oil composition of sesame seeds (Sesamum indicum L.) grown in the Gizan area of Saudi Arabia. Int J Food Sci Nutr 49: 409-414.

Baydar H, Turgut K. 1999. Variation of certain characters and line selection for yield, oleic and linoleicacid in the Turkish sesame (Sesamum indicum L.) populations. J Agr For 23: 431-441.

Borchani C, Besbes S, Blecker C, Attia H. 2010. Chemical characteristics and oxidative stability of sesame seed, sesame paste and olive oils. J Agric Sci Tech 12: 585-596.

Boureima S, Diouf M, Cissé N. 2010. Besoins en eau, croissance et rendement du sésame en zone semi-aride. Agron Afr 22(2): 139-147.

Brody T. 1994. Nutritional Biochemistry, 2nd ed. San Diego, CA: Academic Press, pp 761-794.

Bukya A, Vijayakumar TP. 2013. Properties of industrial fractions of sesame seed (Sesamum indicum L.). Int J Agric Food Sci 3(3): 86-89.

Burden D. 2005. Sesame profile.
Dang TL, Tu G, Tu N, Le TT. 1997. Use of sesame powder to prepare food for babies at 12-36 months of age. J Prev Med 7(4): 78-80.

Dar AA, Arumugam N. 2013. Les lignanes de sésame : les méthodes de purification, les activités biologiques et la biosynthèse-une revue. Bioorg Chem 50: 1-10.

Deme T, Haki GD, Retta N, Woldegiorgis A, Geleta M. 2017. Mineral and anti-nutritional contents of Niger seed (Guizotia abyssinica (L.f.) Cass., Linseed (Linumusita tissimum L.) and Sesame (Sesamum indicum L.) Varieties grown in Ethiopia. Foods 6(27). DOI: $10.3390 /$ foods6040027.

Deosthale YG. 1981. Trace element composition of common oilseeds. J Am Chem Soc 58: 988-990.

Dernekbasi S, Karayücel İ, Parlak Akyüz A. 2017. Evaluation of sesame (Sesamum indicum) seed meal as a replacer for soybean meal in the diets of rainbow trout (Oncorhynchusmy kiss Walbaum, 1792). J Fish Aquat Sci 34(1): 31-39. DOI: 10.12714/ egejfas.2017.34.1.05.

El Asri M, Essahat A, Bouniols A, Mondiès M. 2000. Rendement et qualité des graines du tournesol cultivé sous contrainte hydrique. Résultats des essais en coopération au Maroc et dans le Sud-ouest de la France. In: Proc. XV International Sunflower Conference, Toulouse, France, I C, pp.127-132.

El Khier MKS, Ishag KEA, Yagoub AEA. 2008. Chemical composition and oil characteristics of sesame seed cultivars grown in Sudan. J Agric Biol Sci 4(6): 761-766.

Elleuch M, Besbes S, Roiseux O, Blecke CH, Attia. 2007. Quality characteristics of sesame seeds and by-products, Food Chem 103: 641-650.

Flagella Z, Rotunno T, Di Caterina R, De Simone G, Ciciretti L De Caro A. 2000. Effect of supplementary irrigation on seed yield and oil quality of sunflower (Helianthus annuus L.) grown in a sub-arid environment. In: Proc. XV International Sunflower Conference, Toulouse, France, I C, 139-144.

Gadade BV, Kachare DP, Satbhai RD, Naik RM. 2017. Nutritional composition and oil quality parameters of sesame (Sesamum indicum L.) genotype. Int Res J Multidiscip Stud 3(7): 2454-8499.

Gandhi AP, Srivastava J. 2007. Studies on the production of protein isolates from defatted sesame seed (Sesamum indicum L.) flour and their nutritional profile. ASEAN Food J 14: 175-180.

Gharby S, Hchman H, Bouzoubaa Z, Ali A, Yadini AE, Charrouf Z. 2015. Chemical characterization and oxidative stability of seeds and oil of sesame grown in Morocco. J Saudi Soc Agric Sci. DOI: 10.1016/j.jssas.2015.03.004.

Gopalan C, Ramasastri BV, Balasubramanian SC. 2011. Nutritive value of Indian foods: National Institute of Nutrition, Indian Council of Medical Research, Hyderabad, India Revised Ed 1989; Reprinted 2011.

Hahm TS, Park SJ, Martin Lo Y. 2009. Effects of germination on chemical composition and functional properties of sesame (Sesamum indicum L.) seeds. Bioresour Technol 100: 1643-1647.

Hassan Manal AM. 2012. Studies on Egyptian sesame seeds (Sesamum indicum L.) and its products 1-physicochemical analysis and phenolicacids of roasted Egyptian sesame seeds (Sesamum indicum L.). World J Dairy Food Sci 7(2): 195-201.

Jimoh WA, Fagbenro OA, Adeparusi EO. 2011. Effect of processing on some minerals, anti-nutrients and nutritional composition of sesame (Sesamum indicum) seed meals. EJEAFChe 10(1): 1864.

Kahyaoglu T, Kaya S. 2006. Modelling of moisture, color and texture changes in sesame seeds during the conventional roasting. J Food Eng 75: 167-177.

Kanu PJ, Kerui Z, Ming ZH, Haifeng Q, Kanu JB, Kexue Z. 2007. Sesame protein: Functional properties of sesame (Sesamum indicum $\mathrm{L}$.) protein isolate as influenced by $\mathrm{pH}$, temperature, time 
and ratio of flour to water during its production. Asian J Biochem 2: 289-301. DOI: 10.3923/ajb.2007.289.301. Available from http://scialert.net/abstract/?doi=ajb.2007.289.301.

Kanu PJ. 2011. Biochemical analysis of black and white sesame seeds from China. Am J Biochem Mol Biol 1(2): 145-157. DOI: 10.3923/ajbmb.2011.145.157. Available from http://scialert.net/ abstract/?doi=ajbmb.2011.145.157.

Kim JH, Seo WD, Lee SK, Lee YB, Parka CH, Ryu HW, Lee JH. 2014. Comparative assessment of compositional components, antioxidant effects, and lignan extractions from Korean white and black sesame (Sesamum indicum L.) seeds for different crop years, J Funct Foods 7: 495-505.

Lee TTT, Wang MMC, Hou RCW, Chen LJ, Su RC, Wang CS, Tzen JTC. 2003. Accumulation of sesame $2 \mathrm{~S}$ albuminen hances methionine and cysteine levels of transgenic rice seeds. Biosci Biotechnol Biochem 67: 1699-1705.

Makinde FM, Akinoso R. 2013. Nutrient composition and effect of processing treatments on anti-nutritional factors of Nigerian sesame (Sesamum indicum Linn) cultivars. Int Food Res J 20(5): 2293-2300.

Makinde FM, Akinoso R. 2014. Comparison between the nutritional quality of flour obtained from raw, roasted and fermented sesame (Sesamum indicum L.) seed grown in Nigeria. Acta Sci Pol Technol Aliment 13(3): 309-319.

Mohammed MI, Hamza ZU. 2008. Physicochemical properties of oil extracts from Sesamum indicum (L.) seeds grow in Jigawa state-Nigeria. J Appl Sci Environ Manage 12: 99-101.

Nwaga D, Omoloko C, Nfonfu A, Kengni E, Titanji VPK. 2000. Caractérisation des protéines de réserve des graines d'arachide (Arachis hypogea L) et du niébé (Vigna unguiculata L. walp.). Agron Afr 12(3): 115-126.

Nzikou JM, Matos L, Bouanga-Kalou G, Ndangui CB, Pambou-Tobi, NPG, Kimbonguila A, Silou T, Linder M, Desobry S. 2009. Chemical composition on the seeds and oil of sesame (Sesamum indicum L.) grown in Congo-Brazzaville. Adv J Food Sci Technol 1(1): 6-11.

Nzikou JM, Mvoula-Tsiéri M, Ndangui CB, Pambou-Tobi NPG, Kimbonguila A, Loumouamou B, Silou T, Desobry S. 2010. Characterization of seeds and oil of sesame (Sesamum indicum L.) and the kinetics of degradation of the oil during heating. Res $J$ Appl Sci Eng Technol 2(3): 227-232.

Ogbonna PE, Ukaan SI. 2013. Chemical composition and oil quality of seeds of sesame accessions grown in the Nsukka plains of South Eastern Nigeria. Afr J Agric Res 8(9): 797-803.

Olowe VIO, Adeyemo YA, Adeniregun OO. 2009. Sesame: the under exploited organic oil seed crop. J Sci Sustain Develop 2: 29-32.
Orruno OE, Morgan MRA. 2007. Purification and characterization of the $7 \mathrm{~S}$ globulin storage protein from sesame (Sesamum indicum L.). Food Chem 100: 926934.

Ozkan A, Deniz C, Muhittin K. 2012. Morphological properties and chemical compositions of some sesame (Sesamum indicum L.) populations cultivated in Kilis, Turkey, Afr J Agric Res 7(19): 3029-3033.

Pellett PL, Shadarevian S. 1970. Food composition, tables for use in the Middle East, 2nd ed. Beirut: American University of Beirut.

Peter KV. 2004. Woodhead Publishing in Food Science and Technology: Handbook of Spices. Ed. 2004, Chap. 2. Cambridge.

Prakash K, Naik SN. 2014. Bioactive constituents as a potential agent in sesame for functional and nutritional application. J Bioresour Eng Technol 1: 48-66.

Prasad. 2002. Text book of field crop production. New Delhi: ICAR, pp. 1-821.

Rondanini D, Savin R, Hall AJ. 2003. Dynamics of fruit growth and oil quality of sunflower (Helianthus annuus L.) exposed to brief intervals of high temperature during grain filling. Field Crops Res 83: 79-90.

Russell EW. 1973. Soil conditions and plant growth, 10th ed. Great Britain: Longman.

Sabah El Khier MK, Khogali Elnur AI, Abu El Gasim AY. 2008. Chemical composition and oil characteristics of sesame seed cultivars grown in Sudan. Res J Agric Biol Sci 4(6): 761-766.

Sene B, Sarr F, Sow MS, Diouf D, Niang M, Traoré D. 2017. Physicochemical composition of the sesame variety (Sesamum indicum L.) 32-15 and characterization of its derived products (seeds, oil and oilcake) in Senegal. Food Sci Qual Manag 65 (ISSN 2224-6088 [Paper] ; ISSN 2225-0557 [Online]).

Sher H, Al-Yemeni M, Bahkali AHA, Sher H. 2010. Effect of environmental factors on the yield of selected mushroom species growing in two different agro ecological zones. Saudi J Biol Sci 17: 321-326. DOI: 10.1016/j.sjbs.2010.06.004.

Unal MK, Yalcsn H. 2008. Proximate composition of Turkish sesame seeds and characterization of their oils. Grasas y Aceites 59: 23-26.

Yahya A. 1998. Salinity effects on growth and on uptake and distribution of sodium and some essential mineral nutrients in sesame. J Plant Nutr 21: 7, 1439-1451. DOI: 10.1080/ 01904169809365494.

Yogranjan, Satpute GK, Marabi RS, Mishra MK, Mishra SP. 2014. Global resurgence of sesame (Sesamum indicum L.) utilization: acurrent scenario. Indo-Am J Agric Vet Sci 2(3): 2321-9602.

Zebib H, Bultosa G, Abera S. 2015. Physico-chemical properties of sesame (Sesamum indicum L.) varieties grown in Northern Area, Ethiopia. Agric Sci 6: 238-246. DOI: 10.4236/as.2015.62024.

Citation de l'article : Sene B, Sarr F, Diouf D, Kane A, Traore D. 2018. Étude de la composition minérale et des teneurs en protéines et en matières grasses de huit variétés de sésame (Sesamum indicum L.) introduites au Sénégal pour un criblage variétal. OCL 25(6): A601. 